\title{
Women's experiences of planning a home birth with maternity care providers in middle to high-income countries: a systematic review protocol
}

Maria Healy ( $\square$ Maria.Healy@qub.ac.uk)

Queen's University Belfast Faculty of Medicine Health and Life Sciences https://orcid.org/0000-00033729-7285

\section{Olufikayo Bamidele}

Queen's University Belfast School of Nursing and Midwifery / University of Hull Academy of Primary Care

\section{Patricia Gillen}

Southern Health and Social Care Trust / Ulster Univeristy

\section{Protocol}

Keywords: Home birth, women, planned, childbirth, delivery, maternity care provider, experiences

Posted Date: July 8th, 2020

DOl: https://doi.org/10.21203/rs.3.rs-40587/v1

License: (c) (i) This work is licensed under a Creative Commons Attribution 4.0 International License. Read Full License 


\section{Abstract}

Background: A woman's choice of birth place does not only influence her birth experience, but also impacts on maternal and neonatal outcomes. For healthy women who have had a straightforward pregnancy, a planned home birth supported by midwives and other maternity care providers, is now a recognised choice within their individual country's health care system. However, there is limited evidence on women's actual experiences of engaging with maternity care providers to plan for a home birth, especially within the context of middle to high-income countries where there is integration of maternity care services. Therefore, this systematic review will synthesise findings from previous studies, which have reported on women's experiences of planning a home birth in consultation with maternity care providers, in middle to high-income countries.

Methods: Using a systematic approach, we will develop a search strategy to identify relevant research studies on women's experiences of planning a home birth, with the support of their maternity care providers. Search terms will be iteratively developed using text words derived from the review aim, the PICO framework and database-indexed terms. In May 2020, the searches will be conducted on seven bibliographic databases: Ovid Medline, Embase, Psyclnfo, and CINAHL plus, Scopus, ProQuest and Cochrane (Central and Library) from January 2015 to $26^{\text {th }}$ May 2020. Supplementary searches will also be undertaken to identify additional articles. At least two reviewers will do the screening, quality appraisal, data extraction and analysis. Included studies will be appraised using a quality appraisal tool suited to the study design. Data will be analysed using either a narrative or thematic synthesis depending on the methodological design of the studies included.

Discussion: Review findings will provide useful recommendations to improve care and support provided for women when planning a home birth. We will publish review findings in a peer-reviewed journal and present it at relevant conferences while also sharing summaries with maternity care providers and service users via social media fora.

PROSPERO registration ID: CRD42018095042

\section{Background}

A woman's chosen place of birth impacts not only the type of birth, but also the number of unnecessary interventions that the mother and baby are exposed to during their labour and birth ${ }^{1-4}$. Women who give birth in a midwife-led unit or at home, rather than an obstetric unit, experience lower rates of unnecessary interventions. These include amniotomy, augmentation of labour, instrumental vaginal birth, Caesarean Section, and opiate or regional analgesia ${ }^{5-7,4}$. There is also evidence that the outcomes for both multiparous and nulliparous women and babies of multiparous women who have birthed at home are equal to, if not better than those in other birth settings, for example women are less likely to experience 3 rd or 4 th degree perineal trauma, maternal infection or postpartum haemorrhage ${ }^{4-5,8-9}$. The Birthplace study also reported an increased incidence of adverse outcomes for the baby of nulliparous women who 
had a planned home birth ${ }^{4}$. However, cohort studies on home birth have identified perinatal outcomes from home birth as low and not significantly different for infants of nulliparous women ${ }^{2,10}$.

$\mathrm{NICE}^{8}$, continues to support a policy of offering all women with straightforward pregnancies a choice of birth settings including home, midwifery units (both alongside and freestanding) or obstetric unit). Coxon et $\mathrm{al}^{11}$ conducted a qualitative synthesis of women's decision-making for a birthplace preference and choice. The review identified that women's choice of birthplace were influenced by how informed they were about available options, their right to choose, experiences of previous births, risk perceptions, safety concerns and their care-givers' views (including family, friends and healthcare professionals). Planning birth at home can be enabled by following an evidence-based guideline and co-produced resources for women and their partners ${ }^{12}$.

A position statement on home birth by the International Confederation of Midwives ${ }^{13}$ states that women have a right to home birth as a valid and safe option (p. 1). They also state that women have a right to make an informed decision to give birth at home supported by a midwife within their own countries health care system. A recent joint statement by the Royal College of Midwives and Royal College of Obstetricians and Gynaecologists (RCOG) ${ }^{14}$ asserts that healthy women with low risk pregnancy may benefit from giving birth at home during the evolving Coronavirus (COVID-19) pandemic.

In a principle-based concept analysis, Beecher et al ${ }^{15}$ propose a theoretical definition of 'Women's experiences of their maternity care' as a complex concept referring to women's interpretation of their care encounters within the maternity services. It is subjective in nature and evolves throughout the course of pregnancy, childbirth and the postpartum period. It is dependent upon a woman's individual needs and expectations, shaped by their personal circumstances and influenced by how their care is organised and delivered.'(p. 4). In their systematic review and meta-analysis, Rietsma et al ${ }^{5}$ identified that women who plan to birth at home may hold different values around birth outcomes. However, they also recognised that those who plan a home birth are less likely to experience unnecessary interventions and adverse birth outcomes.

Little is known regarding women's actual experiences of engaging with maternity care providers to plan for a home birth. The dynamics of the woman-healthcare provider relationship in planning for a home birth within the context of a middle to high-income country where women have access to an integrated community and hospital maternity care system, is worthy of investigation. Previous reviews have looked at maternal and neonatal outcomes ${ }^{16-18,1}$ and comparison between planned hospital and planned home births ${ }^{19-20}$. Others have examined post-partum issues ${ }^{21}$, model of care for childbearing women ${ }^{22}$, integration of home birth into a healthcare system ${ }^{23}$ and scope of hospital transfers during homebirth ${ }^{24-}$ 25 . A recent review by Hill ${ }^{26}$ looked at women's experiences of planning a home birth. This review did not follow systematic review methodology and included four papers. A systematic review of studies on women's experiences of planning a home birth is needed to provide an in-depth understanding of what matters to women, including their information and support needs. Insights gleaned from such a 
systematic review could potentially help to enhance woman-healthcare provider interactions in planning for a home birth and inform future service provision to maximise positive experiences for women, planning to birth their babies at home. Therefore, the aim of this systematic review is to synthesise findings from previous studies, which have reported on women's experiences of planning a home birth in consultation with maternity care providers in middle to high-income countries.

\section{Methods}

An important starting point for any review is operational definitions of the concepts under review. Given the rise in literature reporting on unassisted or free birthing, on Babies Born before Arrival (BBA's) to hospital, and the increased visibility of birthing supported by unregistered attendants, operational definitions of planned home birth and maternity care providers are central.

\section{Operational definition of terms \\ We define:}

\section{Planned home birth}

as an informed decision by women to birth their baby at home with the support of maternity care providers.

\section{Maternity care providers}

as healthcare providers involved in supporting women to plan their birth at home. These will include midwives, obstetricians, general practitioners, (G.Ps) anesthetists, paediatricians, and paramedics.

\section{Rationale for focus on planned homebirth in middle- and high-Income Countries}

The organisation of health care differs between countries and between low and middle-income countries and middle and high-income countries. The focus of this review is on middle and high-income countries. The classifications are provided below.

\section{Country Classification}

According to the World Bank classification ${ }^{27}$, high-income countries (also known as developed countries) are countries with per capital gross national income (GNI) of at least $\$ 12,476$ as of 2018. For example, Argentina, Australia, Barbados, Canada, Chile, Croatia, Denmark, New Zealand, France, Germany, Finland, Portugal, Spain, Sweden, Switzerland, United Kingdom, United States of America etc. Middleincome countries have per capita GNI between $\$ 1,025$ and $\$ 12,476$ as of 2018. For example, Angola, Bangladesh, China, Cameroon, Ghana, India, Kenya, Indonesia, Nigeria, Pakistan, Philippines, Sri Lanka, 
Sudan, Tunisia, Vietnam, Zambia. Low-income countries are those with GNI per capita of \$1, 025 or less as of 2018. For example, Afghanistan, Benin, Burkina Faso, Burundi, Central African Republic, Chad, Democratic Republic of Congo, Eritrea, Ethiopia, Gambia, Guinea, Guinea Bissau, Haiti, Korea, Liberia, Madagascar, Malawi, Mali, Mozambique, Nepal, Niger, Rwanda, Sierra Leone, Somalia, South Sudan Tajikistan, Syrian Tanzania, Togo and Uganda, Yemen Rep.

\section{Search strategy}

Using a systematic approach ${ }^{28}$, we will develop a search strategy to identify relevant research studies on women's experiences of planning a home birth, with the support of their maternity care providers. Search terms will be iteratively developed using text words derived from the review aim, the PICO framework ${ }^{29}$ (Population, Intervention, Comparison and Outcome) (Table 1) and database-indexed terms. Broadly, search terms will be words related to: (home birth OR childbirth) AND plan AND experience (see appendix 1 for a detailed draft of the Medline search). We will test and refine the search strategy for accuracy on Ovid Medline prior to running it on other databases. This refined search strategy will be utilised on seven bibliographic databases: Ovid Medline, Embase, Psyclnfo, and CINAHL plus, Scopus, ProQuest and Cochrane (Central and Library) from January 2015 to 26th May 2020. We decided on January 2015 as our cut off point for the searches as the publication of the NICE clinical guideline (CG190) on Intrapartum Care for Healthy Women and Babies was December 2014, which advocated for home birth as a choice of place of birth for women. In line with the Peer Review of Electronic Search Strategies (PRESS) guidelines $^{30}$, we will develop the search strategy in consultation with an experienced subject librarian, which will be checked by at least two authors.

We will tailor the refined search terms to each database's indexing requirement. Boolean operators 'AND' and 'OR' will be used to combine search terms as appropriate. We will also use quotation (") and truncation (*) marks to capture possible variations of the search terms on each database. We will further conduct supplementary searches to identify additional articles, which we may have missed during the electronic database searches. This will include back chain referencing of included papers (hand searching of reference lists), consultation with members of the Regulation and Quality Improvement Authority (RQIA) Planning to Birth at Home in Northern Ireland ${ }^{12}$ guideline development group, professional networks and grey literature search (for example, OpenGrey). We will run the searches again on the selected databases prior to the final analysis in order to identify any article newly published since our last search. We will manage search results with the bibliographic databases Endnote, Refworks and Covidence. Deduplication of retrieved articles will be undertaken on Endnote and Covidence using a systematic method ${ }^{31}$. The review is registered on the International Prospective Register of Systematic Reviews (PROSPERO: Registration ID: CRD42018095042) 
Table 1

PICO Framework

Population

Comparator

Outcome(s)

\section{Inclusion Criteria}

- Women who planned or are planning a home birth within the context of a middle or high-income country in consultation with maternity care providers

\section{Exclusion criteria}

-Women who had an unplanned or unassisted/free home birth

- Women planning a home birth without consulting with a professional maternity care provider

- Women who planned or are planning a home birth within the context of a low-income country (low income countries are excluded because their healthcare provision or context is different to that of middle to high-income countries)

Intervention/Exposure

Primary studies which: (1) focused on the planning phase of the home birth experience for women (planned home birth as defined above) and (2) reported on women's experiences of planning their home birth with their maternity care providers

(i) Women's experiences of planning a home birth

(ii) Women's perceptions of their consultation with maternity care providers to plan a home birth

\section{Identification and selection of studies}

Studies will be identified and selected based on the following inclusion and exclusion criteria:

\section{Inclusion criteria}

Primary studies, which investigated women's experiences of planning a home birth within the context of middle and high-income countries, reported in English language and published between January 2015 and May 2020 will be included. Studies that report on women's experience and/or perceptions of their consultation with maternity care providers when planning a home birth will be also be included.

\section{Exclusion criteria}

We will exclude grey literature, which lacks a clear methodology (for example, editorials and books), conference abstracts whose full papers cannot be accessed and PhD and MSc dissertations. We will also exclude studies focused on healthcare professionals' or partners' views on home birth planning. We will exclude home birth studies that lack clear separate data on women's experiences of the planning phase of the home birth, and studies conducted in low-income countries.

\section{Screening}


Following deduplication on Endnote, we will upload the remaining articles into a systematic review management software by Cochrane ${ }^{32}$ to manage the screening process in a rigorous and transparent approach in line with the Preferred Reporting Items for Systematic Reviews and Meta-Analysis guidelines (PRISMA) ${ }^{33}$. At least two reviewers (MH, OB or PG) will independently screen the titles and abstracts) of retrieved studies to remove irrelevant articles. Two authors will resolve any conflicts and if not possible, a third author will review and then all three authors will reach agreement. Two authors ( $\mathrm{MH}, \mathrm{OB}$ or $\mathrm{PG}$ ) will then screen the full text of potentially relevant articles against the review's inclusion and exclusion criteria. We will resolve differences in opinion through discussion (by a minimum of two authors) to reach a mutual agreement. We will report the study selection process on a PRISMA diagram (see Fig. 1).

\section{Quality appraisal}

At least two reviewers ( $\mathrm{MH}, \mathrm{OB}$ or $\mathrm{PG}$ ) will independently appraise the quality of the included studies using an appraisal tool relevant to each study's methodological design. We will appraise studies using the Critical Appraisal Skills Programme (CASP) tool ${ }^{34}$ suited to each study's design. For example, qualitative studies will be assessed using the CASP tool for qualitative studies. We will appraise RCT studies (if included), using the CASP tool for RCTs, although we do not expect to find any RCTs due to the nature of the review question. We will assess other quantitative studies (non-RCTs), using the Grading of Recommendations Assessment, Development and Evaluation (GRADE) tool ${ }^{35}$. Mixed Methods studies will be assessed using the Mixed Methods Appraisal (MMAT) tool ${ }^{36}$. We will assess risk of bias in RCTs (if included) using the Cochrane risk of bias tool ${ }^{37}$ and the Confidence in the Evidence for Reviews of Qualitative Research (CERQual) tool ${ }^{38}$ for qualitative studies.

\section{Data extraction}

At least two reviewers will extract data using a standardised form on MS Excel or MS Word. Conflicts will be resolved through discussion. We will systematically extract data on outcomes related to women's experiences on planning a home birth with their maternity care providers. We will extract data on the study title, author(s) and year of publication, study setting, methodology, population, key findings, quality appraisal score and key conclusions. Where possible, we will attempt to retrieve missing data in relevant studies by contacting the corresponding author.

\section{Data Analysis}

At least two reviewers will analyse aggregate data from the final included studies and resolve any conflict through discussion. The approach for data analysis will be determined by the methodological design of the included studies. If the studies are not sufficiently homogenous (for example, inclusion of a mixture of qualitative and quantitative studies), we will conduct a narrative synthesis. If all studies included are qualitative studies, we will undertake a thematic synthesis ${ }^{39}$. NViVo 12 software will be used to manage the data analysis process where appropriate.

\section{Discussion}


Findings will be discussed in relation to existing research. Review findings will provide useful recommendations to improve the experiences of women planning a home birth. We will publish review

findings in peer-reviewed journal, and present at relevant conferences while also sharing summaries with maternity care providers and service users via social media fora.

\section{Abbreviations}

CASP Critical Appraisal Skilled Programme

CERQual Confidence in the evidence for Reviews of Qualitative Research

GNI Gross National Income

MMAT Mixed Methods Appraisal Tool

MLU Midwifery-Led Unit

NICE National Institute for Health and Care Excellence

PICO Population, Intervention, Comparator and Outcome

PRISMA Preferred Reporting Items for Systematic Reviews and Meta-Analysis

RQIA Regulation and Quality Improvement Authority

RCT Randomised Controlled Trial

\section{Declarations}

\section{Ethical approval and consent to participate:}

This is a protocol for a systematic review, which utilises published data, and therefore ethical approval was not required.

Consent for publication:

Not applicable

Availability of data and materials:

Not applicable

Competing interests:

The authors declare they have no competing interests. 


\section{Funding:}

The Southern Health and Social Care Trust HSC Research and Development Fund and matched funding from Public Health Agency Opportunity-led Research. Ulster University's Institute of Nursing and Health Research funded access to Covidence, which is Cochrane's web-based software platform, which improves the efficiency of producing systematic reviews. The funders were not involved in collection, analysis and interpretation of the data.

\section{Authors' contributions:}

$\mathrm{MH}$ and $\mathrm{PG}$ conceived the review and designed the study. All authors $(\mathrm{MH}, \mathrm{OB}$ and $\mathrm{PG})$ contributed to the writing of the protocol and will contribute to the collection, analysis and interpretation of the data. PG is the guarantor of the review.

\section{Acknowledgements:}

Sincere thanks to Mary Rose Holman (Subject Librarian, Life and Health Sciences, Ulster University) who assisted with the design of the initial search strategy and database searches. In addition, thanks go to members of the RQIA Planning a Home Birth Guideline Development Group who undertook reviews of some papers in our preliminary review during development of the guideline ${ }^{12}$

\section{References}

1. Scarf V, Rossiter C, Vedam S, Dahlen H, Ellwood D, Forster D, et al. Maternal and perinatal outcomes by planned place of birth among women with low-risk pregnancies in high-income countries: A systematic review and meta-analysis. Midwifery. 2018;62:240-55.

2. de Jonge A, Geerts C, van der Goes B, Mol B, Buitendijk S, Nijhuis J. Perinatal mortality and morbidity up to 28 days after birth among 743070 low-risk planned home and hospital births: a cohort study based on three merged national perinatal databases. BJOG: An International Journal of Obstetrics Gynaecology. 2015;122(5):720-8.

3. Davis D, Baddock S, Pairman S, Hunter M, Benn C, Wilson D, et al. Planned place of birth in New Zealand: does it affect mode of birth and intervention rates among low-risk women? Birth. 2011;38(2):111-9.

\section{4. $10.1136 /$ bmj.d7400}

Brocklehurst P, Hardy P, Hollowell J, Linsell L, Macfarlane A, McCourt C, et al. (2011) Perinatal and maternal outcomes by planned place of birth for healthy women with low risk pregnancies: the Birthplace in England national prospective cohort study. BMJ. 2011; 23 343:d7400. doi: 10.1136/bmj.d7400. 
5. Reitsma A, Simioni S, Brunton G, Kaufman K, Hutton E. Maternal outcomes and birth interventions amongwomenwhobegin labour intending to give birth at home compared to women of low obstetrical risk who intend to give birth in hospital: A systematic review and meta-analyses. EClinical Medicine 21 (2020) 100319 https://www.thelancet.com/journals/eclinm/article/PIIS25895370(20)30063-8/fulltext. Accessed 12 June 2020.

6. Hutton E, Cappelletti A, Reitsma A, Simioni J, Horne J, McGregor C, Ahmed R. Outcomes associated with planned place of birth among women with low-risk pregnancies. Can Med Assoc J. 2016;188(5):E80-90. https://doi. 10.1503/cmaj.150564.

7. Halfdansdottir B, Smarason A, Olafsdittir O, Hildingsson I, Sveinsdottir H. Outcome of planned home and hospital births among low-risk women in Iceland in 2005-2009: A retrospective cohort study. Birth. 2015;42(1):16-26.

8. National Institute of Clinical Excellence (NICE). (2014) Clinical Guideline 190 Intrapartum care: care of the healthy woman and their babies during childbirth. http://www.nice.org.uk/guidance/cg190/resources/guidance-intrapartum-carecare-of-healthywomen-and-their-babies-during-childbirth-pdf. Accessed 18 June 2020.

9. $10.1136 / \mathrm{bmj} . \mathrm{d} 7400$

Hollowell J, Puddicombe D, Rowe R, Linsell L, Hardy P, Stewart M, et al. The Birthplace national prospective cohort study: perinatal and maternal outcomes by planned place of birth. Birthplace in England research programme. Final report part 4. London: NIHR Service Delivery and Organisation programme; 2011. BMJ. 2011; Nov 24. doi: 10.1136/bmj.d7400 https://www.ncbi.nlm.nih.gov/pmc/articles/PMC3223531/.

10. Van der Kooy J, Poeran J, De Graaf J, Birnie E, Denktass S, Steegers E, et al. Planned home compared with planned hospital births in the Netherlands: intrapartum and early neonatal death in low-risk pregnancies. Obstetrics Gynaecology. 2011;118:1037-46.

11. Coxon K, Chisholm A, Malouf R, Rowe R, Hollowell J. What influences birth place preferences, choices and decision-making amongst healthy women with straightforward pregnancies in the UK? A qualitative evidence synthesis using a 'best fit' framework approach. BMC Pregnancy Childbirth. 2017;17(1):103.

12. Regulation and Quality Improvement Authority (RQIA). (2019) Planning to Birth at Home in Northern Ireland https://. Accessed 22 June 2020.

13. International Confederation of Midwives. (2017) Position Statement HomebirthPS2011_010 V2017 ENG https://www.internationalmidwives.org/assets/files/statement-files/2018/04/eng-homebirth14.pdf. Accessed on 10 June 2020.

14. Royal College of Midwives and Royal College of Obstetricians and Gynaecologists. (2020). Coronavirus (COVID-19) Infection in Pregnancy Information for healthcare Professionals Version 10: 4 June London: RCOG. https://www.rcog.org.uk/coronavirus-pregnancy. Accessed on 10 June 2020.

15. 10.1016/j.wombi.2019.11.001 
Beecher C, Devane D, White M, Greene R, Dowling M. Women's experience of their maternity care: a principle-based concept analysis Women and Birth Article in Press. 2019

https://doi.org/10.1016/j.wombi.2019.11.001. Accessed 18 June 2020.

16. Catling-Paull C, Coddington R, Foureur M, Homer C. Birthplace in Australia Study; National Publiclyfunded Homebirth Consortium Publicly funded homebirth in Australia: a review of maternal and neonatal outcomes over 6 years. Med J Aust. 2013;198(11):616-20.

17. Elder H, Alio A, Fisher S. Investigating the debate of home birth safety: A critical review of cohort studies focusing on selected infant outcomes. Japan Journal of Nursing Science: JJNS. 2016;13(3):297-308.

18. Kobayashi S, Hanada N, Matsuzaki M, Takehara K, Ota E, Sasaki H, Nagata C, Mori R. Assessment and support during early labour for improving birth outcomes. Cochrane Database of Systematic Reviews. 2017;4(4):CD011516. https://doi. 10.1002/14651858.CD011516.pub2.

19. Olsen O, Clausen J. Planned hospital birth versus planned home birth. Cochrane database of systematic reviews (Online) 2012;9 (000352).

20. Rossi A, Prefumo F. Planned home versus planned hospital births in women at low-risk pregnancy: A systematic review with meta-analysis. European Journal of Obstetrics Gynecology Reproductive Biology. 2018;222:102-8.

21. Pantoja T, Abalos E, Chapman E, Vera C, Serrano V. Oxytocin for preventing postpartum haemorrhage (PPH) in non-facility birth settings. Cochrane Database of Systematic Reviews. 2016;14:4:CD011491.

22. 10.1002/14651858.CD004667.pub5

Sandall J, Soltani H, Gates S, Shennan A, Devane D. Midwife-led continuity models versus other models of care for childbearing women. Cochrane Database of Systematic Reviews. 2016; Issue 4. Art. No.: CD004667. DOI: 10.1002/14651858.CD004667.pub5.

23. Comeau A, Hutton E, Simioni J, Anvari E, Bowen M, Kruegar S, et al. Home birth integration into the health care systems of eleven international jurisdictions. Birth. 2018;45(3):311-21.

24. Blix E, Kumle M, Kjaergaard H, Oian P, Lindgren H. Transfer to hospital in planned home births: a systematic review. BMC Pregnancy \& Childbirth. 2014; 14.

25. Vedam, SLeeman, L, Cheyney M, Fisher TJ, Myers SKane, Low L, Ruhl C. Transfer from Planned Home Birth to Hospital: Improving Interprofessional Collaboration Journal of Midwifery and Women's Health 59 (6): 624-634 doi:10.1111/jmwh.12251.

26. Hill L. Women's experiences of planned home birth: a review of the literature. MIDIRS Midwifery Digest. 2020;30:1.

27. The World Bank. (2020) World Bank Country and Lending Groups Country Classification. https://datahelpdesk.worldbank.org/knowledgebase/articles/906519. Accessed 1 June 2020.

28. Centre for Reviews and Dissemination. (2009). Systematic reviews: CRD's guidance for undertaking reviews in healthcare. http://www.york.ac.uk/crd/SysRev/!SSL!/WebHelp/SysRev3.htm Accessed 15 June 2020. 
29. Thomas J, Kneale D, McKenzie JE, Brennan SE, Bhaumik S. Chapter 2: Determining the scope of the review and the questions it will address. In: Higgins JPT, Thomas J, Chandler J, Cumpston M, Li T, Page MJ, Welch VA, editors. Cochrane Handbook for Systematic Reviews of Interventions version 6.0 (updated July 2019). Cochrane, 2019. . Accessed 6 December 2019.

30. McGowan J, Sampson M, Salzwedel DM, Cogo E, Foerster V, Lefebvre C. PRESS Peer Review of Electronic Search Strategies: 2015 guideline statement. J Clin Epidemiol. 2016;75:40-6.

31. Bramer W, Giustini D, de Jonge G, Holland L, Bekhuis T. De-duplication of database search results for systematic reviews in EndNote. J Med Libr Assoc. 2016;104(3):240-3.

32. Covidence. (2019) World-class systematic review management. https://www.covidence.org/home Accessed 15 June 2020.

33. PRISMA. (2015) Transparent Reporting of Systematic Reviews and Meta-analysis. http://www.prisma-statement.org/ Accessed 15 June 2020.

34. CASP. (2020) CASP Checklists. https://casp-uk.net/casp-tools-checklists/. Accessed 22 June 2020.

35. GRADE Working Group. Grading quality of evidence and strength of recommendations. BMJ. 2004;328:1490.

36. Hong Q, Fàbregues S, Bartlett G, Boardman F, Cargo M, Dagenais P, et al. The Mixed Methods Appraisal Tool (MMAT) Version 2018 for Information Professionals and Researchers'. Education for Information, 2018; 34; 285-291, 2018.

37. Sterne JAC, Savović J, Page MJ, Elbers RG, Blencowe NS, Boutron I, Cates CJ, Cheng H-Y, Corbett MS, Eldridge SM, Hernán MA, Hopewell S, Hróbjartsson A, Junqueira DR, Jüni P, Kirkham JJ, Lasserson T, Li T, McAleenan A, Reeves BC, Shepperd S, Shrier I, Stewart LA, Tilling K, White IR, Whiting PF, Higgins JPT. RoB 2: a revised tool for assessing risk of bias in randomised trials. BMJ. 2019;366:14898.

38. CERQual. (2018) Confidence in the Evidence from Reviews of Qualitative Research. https://www.cerqual.org/ Accessed 15 June 2020.

39. Thomas J, Harden A. Methods for the thematic synthesis of qualitative research in systematic reviews. BMC Medical research Methodology. 2008; 8(45).

\section{Figures}



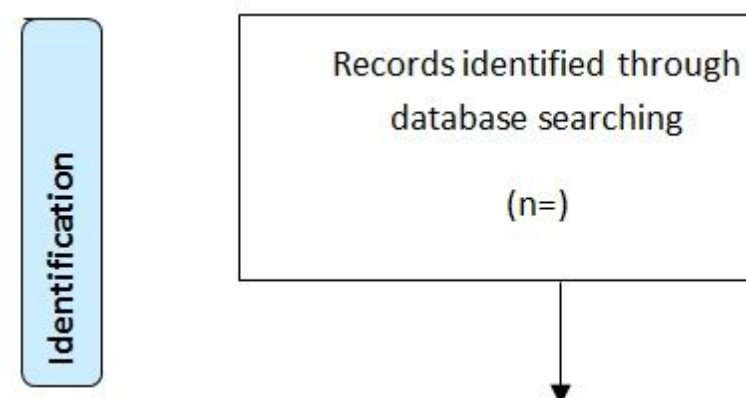

Additional records identified through supplementary search

$(n=)$

$(n=)$

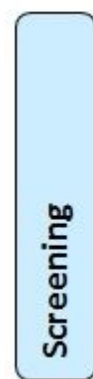

Records after duplicates removed

$(n=)$
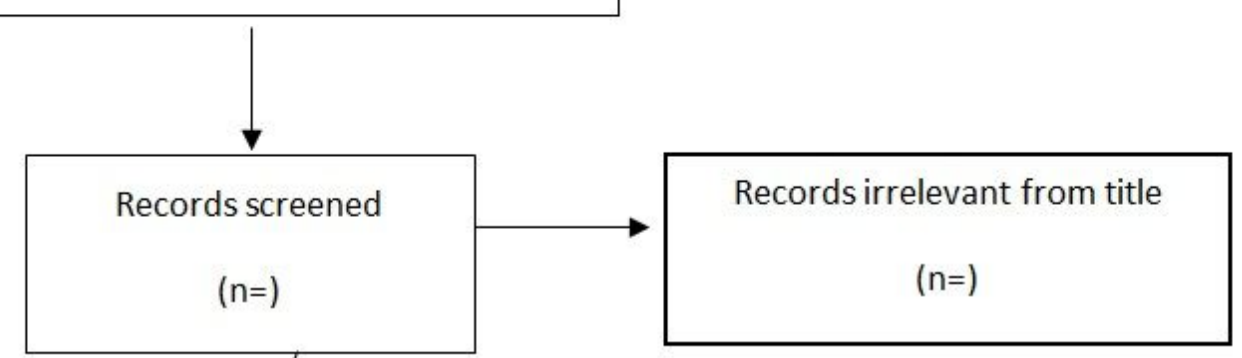

辛
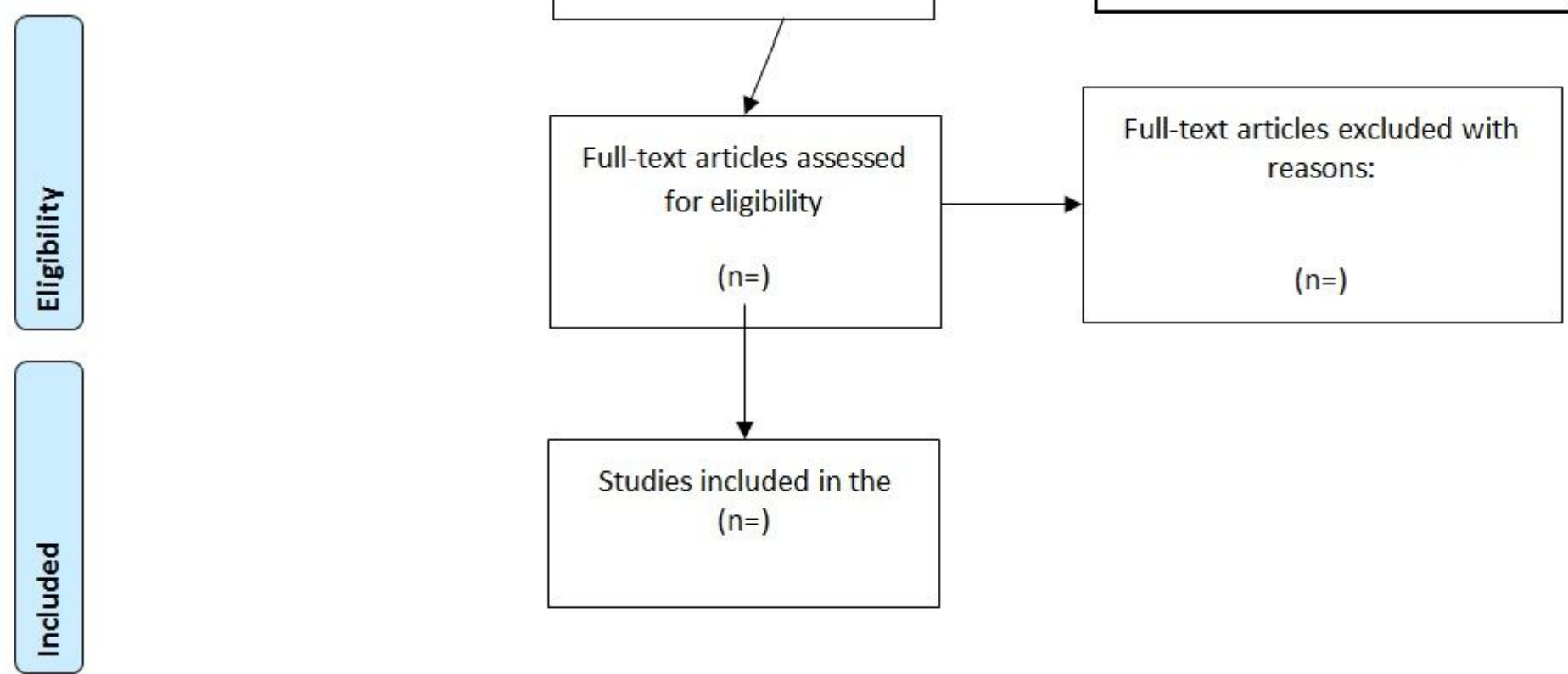

Studies included in the

$(n=)$

\section{Figure 1}

PRISMA flow chart for reporting the search (PRISMA 2015) 